\title{
Plasma marker of tissue oxidative damage and edaravone as a scavenger drug against peroxyl radicals and peroxynitrite
}

\author{
Yorihiro Yamamoto* \\ School of Bioscience and Biotechnology, Tokyo University of Technology, 1404-1 Katakura, Hachioji, Tokyo $192-0982$ Japan
}

(Received 25 July, 2016; Accepted 9 August, 2016; Published online 12 November, 2016)

\begin{abstract}
The percentage of the plasma oxidized form of coenzyme Q10 in the total amount of coenzyme $\mathrm{Q} 10\left(\% \mathrm{CoQ}_{10}\right)$ is a useful marker of oxidative stress in the circulation. Plasma free fatty acids and their composition can be used as markers of tissue oxidative damage, as demonstrated in patients suffering from a wide variety of diseases and in humans and rats under oxidative stress. Edaravone was approved for the treatment of stroke in Japan in 2001 and its mechanism of action is based on scavenging lipid peroxyl radicals. In 2015, edaravone was also approved for the treatment of ALS patients. Edaravone functions therapeutically as a scavenger of peroxynitrite, as demonstrated by the finding that its administration raises plasma uric acid levels and decreases 3-nitrotyrosine in cerebrospinal fluid.
\end{abstract}

Key Words: coenzyme Q10 redox balance, plasma levels of free fatty acids and their composition, edaravone, ALS, peroxynitrite

\section{Plasma Markers of Oxidative Stress}

Reactive oxygen and nitrogen species have been suggested to increase oxidative stress and to cause aging and degenerative diseases such as heart attack, stroke, neurodegenerative diseases, diabetes, and cancer. Oxidative stress is defined as a disturbance in the pro-oxidant-antioxidant balance in favor of pro-oxidants. ${ }^{(1)}$ The redox balance of coenzyme Q10 $\left(\mathrm{CoQ}_{10}\right)$ could be a good marker of oxidative stress because its reduced form (ubiquinol-10, $\mathrm{CoQ}_{10} \mathrm{H}_{2}$ ) is highly reactive with oxygen radicals and is converted to the oxidized form (ubiquinone-10, $\mathrm{CoQ}_{10}$ ). The incubation of human plasma at $37^{\circ} \mathrm{C}$ under aerobic conditions in the presence of $5 \mu \mathrm{M}$ cupric ion resulted first in a decrease in vitamin $\mathrm{C}$ (VC; ascorbic acid), ${ }^{(2)}$ followed by a rapid decrease in $\mathrm{CoQ}_{10} \mathrm{H}_{2}$ and concomitant production of an equal amount of $\mathrm{CoQ}_{10}$ (Fig. 1). Interestingly, no significant decrease in vitamin $\mathrm{E}$ (VE) was observed despite VE being a self-sacrificing strong antioxidant against lipid peroxidation (eqs. 1 and 2). However, when the lipid peroxyl radical (LOO') concentration is much smaller than the VE concentration, equation 2 does not hold and the VE radical (VE') abstracts a proton from lipid to give lipid radical ( $\mathrm{L}^{\circ}$ ) and VE (eq. 3$),{ }^{(3)}$ although this reaction is very slow. The coupling of eqs. 1,3 , and 4 creates a new cycle (tocopherol-mediated peroxidation) in which lipid hydroperoxide ( $\mathrm{LOOH}$ ) accumulates without any loss of VE, as observed in the second half (after $40 \mathrm{~min}$ ) of plasma oxidation (Fig. 1).(2) The large amount of cholesterol ester in plasma supports the formation of its hydroperoxide (CE-OOH) (Fig. 1).

$$
\mathrm{LOO}^{*}+\mathrm{VE} \rightarrow \mathrm{LOOH}+\mathrm{VE}^{*}
$$

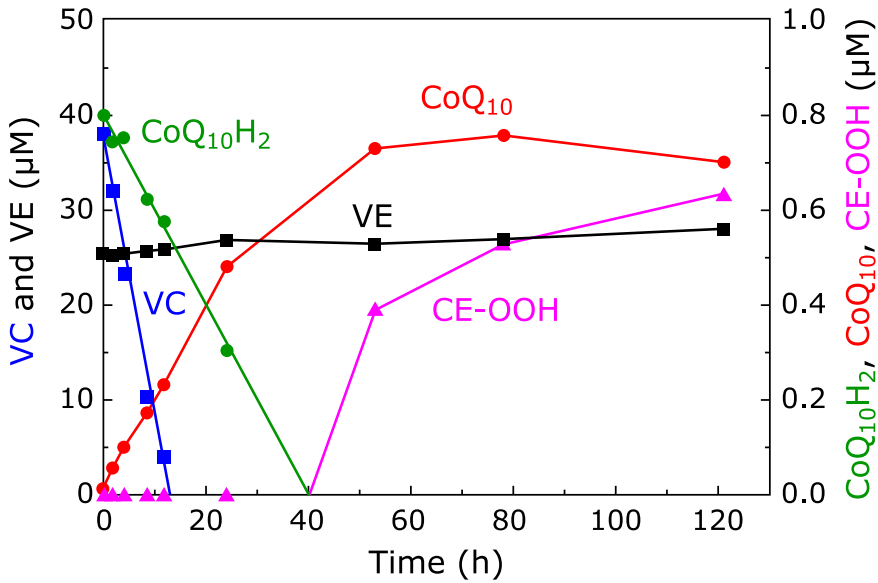

Fig. 1. Changes in plasma antioxidant levels during the aerobic oxidation of human plasma in the presence of $5 \mu \mathrm{M}$ cupric chloride at $37^{\circ} \mathrm{C}$. VC, ascorbic acid; $\mathrm{CoQ}_{10} \mathrm{H}_{2}$, reduced form of coenzyme Q10; CoQ10, oxidized form of coenzyme Q10; VE, vitamin $\mathrm{E} ; \mathrm{CE}-\mathrm{OOH}$, cholesterol ester hydroperoxide.

$$
\begin{aligned}
& \mathrm{VE}^{*}+\mathrm{LOO}^{*} \rightarrow \mathrm{VE}-\mathrm{OOL} \\
& \mathrm{VE}^{\bullet}+\mathrm{LH} \rightarrow \mathrm{VE}+\mathrm{L}^{\bullet} \\
& \mathrm{L}^{\cdot}+\mathrm{O}_{2} \rightarrow \text { LOO }^{*}
\end{aligned}
$$

The above results indicate that the percentage of $\mathrm{CoQ}_{10}$ in total plasma $\left(\% \mathrm{CoQ}_{10}\right)$ could be a good marker of early stage oxidative stress. We therefore developed a simple and reliable method for the simultaneous detection of plasma $\mathrm{CoQ}_{10} \mathrm{H}_{2}$ and $\mathrm{CoQ}_{10}$ and applied the method to patients with various diseases, ${ }^{(4)}$ as well as to humans and rats under oxidative stress conditions (Table 1). Significant increases in $\% \mathrm{CoQ}_{10}$ were observed in patients with hepatitis, ${ }^{(5)}$ cirrhosis, ${ }^{(5)}$ hepatoma,${ }^{(5)}$ juvenile fibromyalgia,${ }^{(6)}$ amyotrophic lateral sclerosis (ALS), ${ }^{(7)}$ post cardiac arrest syndrome (manuscript in preparation), and heart attack (manuscript in preparation) as compared to age-matched healthy controls. It is interesting that newborn babies have significantly higher plasma $\% \mathrm{CoQ}_{10}$ than adults. ${ }^{(8)}$ Plasma $\% \mathrm{CoQ}_{10}$ increases in triathlon athletes the day after a race (manuscript in preparation). Long-

*To whom correspondence should be addressed.

E-mail: junkan@stf.teu.ac.jp

He received "The SFRR Japan Prize" in 2015 in recognition of his outstanding work. 
Table 1. Changes in plasma markers of oxidative stress and cellular oxidative damage in patients with various diseases as compared to healthy controls and humans or rats under oxidative stress conditions

\begin{tabular}{|c|c|c|c|c|c|c|c|}
\hline Diseases or conditions & $\% \operatorname{CoQ}_{10}(9)$ & Ref & FFA & $\%$ PUFA & $\% 16: 1$ & $\% 18: 1$ & Ref \\
\hline Hepatitis, cirrhosis, and hepatoma & $\uparrow$ & $(5)$ & $\uparrow$ & $\downarrow$ & $\uparrow$ & $\uparrow$ & in prep \\
\hline Juvenile fibromyalgia & $\uparrow$ & (6) & $\uparrow$ & $\downarrow$ & $\uparrow$ & $\uparrow$ & (6) \\
\hline ALS & $\uparrow$ & (7) & NS & $\downarrow$ & NS & NS & (7) \\
\hline Post-cardiac arrest syndrome & $\uparrow$ & in prep & $\uparrow$ & $\downarrow$ & NS & NS & in prep \\
\hline Heart attack & $\uparrow$ & in prep & $\uparrow$ & NS & $\uparrow$ & NS & in prep \\
\hline Newborn babies at day 1 & $\uparrow$ & (8) & $\uparrow$ & $\downarrow$ & $\uparrow$ & NS & (8) \\
\hline Right after a triathlon race & NS & in prep & $\uparrow$ & $\downarrow$ & $\uparrow$ & $\uparrow$ & in prep \\
\hline 1 day after a triathlon race & $\uparrow$ & in prep & NS & $\downarrow$ & $\uparrow$ & $\uparrow$ & in prep \\
\hline LEC rat at 24 weeks of age with trientine treatment & $\downarrow$ & (9) & NS & $\uparrow$ & $\downarrow$ & $\downarrow$ & (9) \\
\hline Rat, chronic $\mathrm{CCl}_{4}$ poisoning & & & NS & $\downarrow$ & $\uparrow$ & $\uparrow$ & $(10)$ \\
\hline Rat, single $\mathrm{CCl}_{4}$ poisoning & NS & in prep & $\uparrow$ & $\downarrow$ & $\uparrow$ & $\uparrow$ & in prep \\
\hline Rat, brain attack model & NS & (11) & $\uparrow$ & NS & $\uparrow$ & $\uparrow$ & (11) \\
\hline Above rat with edaravone treatment & NS & (11) & NS & NS & $\downarrow$ & $\downarrow$ & (11) \\
\hline
\end{tabular}

Ref, reference; in prep, manuscript in preparation.<smiles>C=COc1cc(C)nn1-c1ccccc1</smiles>

Enol form<smiles>C=CC1CC(C)=NN1c1ccccc1</smiles>

Keto form<smiles>Cc1cc(=O)n(-c2ccccc2)[nH]1</smiles>

Amine form<smiles>Cc1cc([O-])n(-c2ccccc2)n1</smiles>

Edaravone anion<smiles>CC1=NN(c2ccccc2)C(=O)C1</smiles>

Edaravone radical

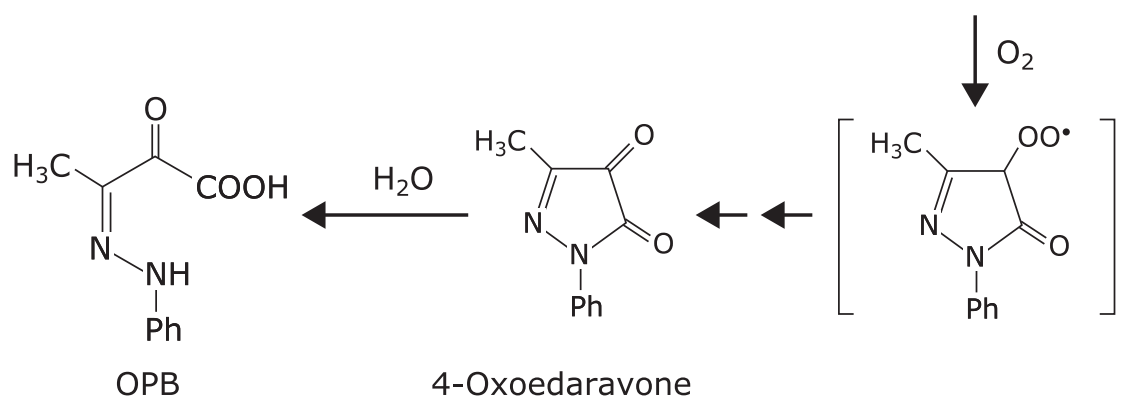

Fig. 2. Pathways for the reduction of lipid peroxyl radicals (LOO*) by the edaravone anion and formation of its oxidation products, 4-oxoedaravone and $\mathrm{OPB}$.

Evans cinnamon (LEC) rats accumulate copper, especially in the liver, and develop hepatitis by 24 weeks of age. Treatment with trientine, a copper chelator, prevents the onset of hepatitis and an increase in plasma percent coenzyme Q9 $\left.\left(\% \mathrm{CoQ}_{9}\right)\right)^{(9)}$

However, an elevation of plasma $\% \mathrm{CoQ}_{10}$ does not reflect oxidative damage inside a specific tissue because plasma $\mathrm{CoQ}_{10}$ is located in circulating lipoproteins. For example, no significant elevation of plasma $\% \mathrm{CoQ}_{9}$ was observed in $\mathrm{CCl}_{4}$-poisoned rats because $\mathrm{CCl}_{4}$ is metabolized to trichloromethylperoxyl radical $\left(\mathrm{Cl}_{3} \mathrm{COO}\right)^{\circ}$ in the liver. ${ }^{(10)}$ Moreover, rats with a middle cerebral artery occlusion did not show any increase in plasma $\% \mathrm{CoQ}_{9}{ }^{(11)}$

\section{Plasma Marker of Tissue Oxidative Damage}

As discussed above, $\% \mathrm{CoQ}_{10}$ is useful for evaluating the formation of oxygen radicals in blood plasma, but it would be more 

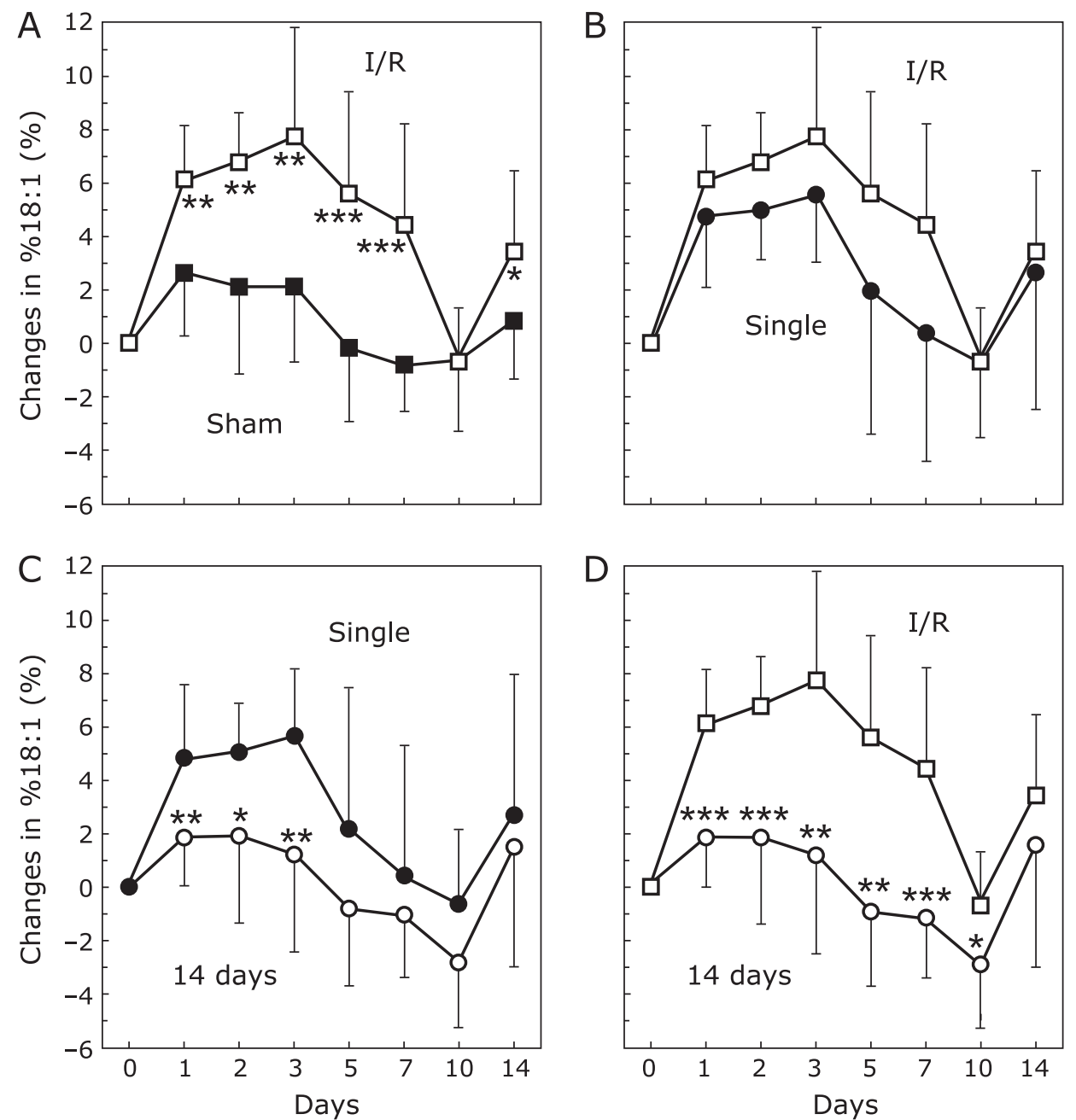

Fig. 3. Time course of changes in plasma content of oleic acid in the total free fatty acids (\%18:1) after $2 \mathrm{~h}$ occlusion of the rat middle cerebral artery and reperfusion $(I / R ; \square)$ or without occlusion (sham; $\mathbf{\square})$. Edaravone $(3 \mathrm{mg} / \mathrm{kg}$ ) was given intravenously immediately after reperfusion (single; ) or twice a day for 2 weeks (14 days; $\bigcirc$ ). Values at day 0 before occlusion were used as baseline. Points and bars show the mean \pm SD. * $p<0.05$, $* * p<0.01$ and $* * * p<0.001$, significant differences between the two groups as determined by Student's $t$ test.

practical to have a plasma marker of tissue oxidative damage. We focused on plasma total free fatty acids (FFAs) because the activities of phospholipase $A_{2}$ and $A_{1}$ increase under oxidative stress and the resulting FFAs may enter the bloodstream through leakage or lysis of oxidatively damaged tissues. ${ }^{(12-14)}$ If this were indeed the case, we would expect an increase in plasma FFA concentration and a decrease in polyunsaturated fatty acids (PUFAs) such as linoleic acid (18:2), linolenic acid (18:3), arachidonic acid (20:4) and docosahexaenoic acid (22:6) in the blood plasma since they are highly susceptible to oxidation. ${ }^{(15)}$ The oxidative loss of PUFAs should be compensated by an increase in monoenoic acids such as palmitoleic acid (16:1) and oleic acid (18:1) due to the action of stearoyl-CoA desaturase. ${ }^{(16)}$ Table 1 shows that plasma FFA levels and the percentages of $16: 1$ and 18:1 in total FFA (\%16:1 and $\% 18: 1$, respectively) were elevated and that the percentages of PUFAs in total FFA (\%PUFA) decreased in all diseases and oxidative stress conditions studied (Table 1).

\section{Free Radical Scavenger Drug}

Table 1 shows that trientine and edaravone (3-methyl-1-phenyl2-pyrazolin-5-one) ameliorated oxidative damage in rats induced by copper and middle cerebral artery occlusion, ${ }^{(9,11)}$ respectively, suggesting that an appropriate drug should be effective for treating human diseases. Many companies have attempted to develop a reactive oxygen scavenging drug.

In late 1980s, Mitsubishi Chemical Corporation was developing an antioxidant as a drug for the treatment of stroke, and animal experiments indicated that edaravone was the most promising lead. ${ }^{17,18)}$ In 1990, I was asked to elucidate the mechanism by which edaravone prevents lipid peroxidation. ${ }^{(19)}$ The $\mathrm{pKa}$ value of edaravone is 7.0 , and the rate of edaravone oxidation initiated by an azo compound increases with increasing $\mathrm{pH}$, suggesting that the anionic form of edaravone is more reactive than the nondissociated form. Donation of an electron to a lipid peroxyl radical converts edaravone anion to the edaravone radical, which is oxidized to produce 3-methyl-1-phenyl-2-pyrazolin-4,5-dione (4-oxoedaravone) and its hydrolysate, 2-oxo-3-(phenylhydrazono) butanoic acid (OPB), as major products of peroxyl radical-induced oxidation (Fig. 2). ${ }^{(19)}$ Edaravone inhibits lipid peroxidation as efficiently as do well-known antioxidants such as VE and VC. Furthermore, a combination of edaravone with VC or VE almost completely inhibits the oxidation of phosphatidylcholine liposomal membrane by lipid-soluble and water-soluble azo initiators. ${ }^{(19)}$

Intravenous injection of edaravone at a dose of $30 \mathrm{mg}$ twice a day for 14 days in patients with acute ischemic stroke, commencing 
within $24 \mathrm{~h}$ after onset, significantly improved the functional outcome in a multicenter, randomized, placebo-controlled, double-blind study. ${ }^{(20)}$ Accordingly, in 2001 the Japanese Ministry of Health, Labor, and Welfare approved intravenous injection of $30 \mathrm{mg}$ edaravone twice a day for a maximum of 14 days for patients with acute brain infarction within $24 \mathrm{~h}$ after onset, and edaravone is now widely used in Japan for the treatment of acute stroke.

To obtain direct evidence that edaravone serves as an antioxidant in vivo, four groups of rats were prepared: an ischemia/ reperfusion (I/R) group receiving $2 \mathrm{~h}$ occlusion-reperfusion of the middle cerebral artery, a single administration group treated by intravenous injection of edaravone $(3 \mathrm{mg} / \mathrm{kg})$ immediately after $\mathrm{I} / \mathrm{R}$, a repeated treatment group receiving twice daily edaravone administrations for 14 days, and a sham operation group without occlusion. ${ }^{(11)}$ Repeated treatment with edaravone significantly improved the neurological symptoms and decreased the impairment of motor function as compared to the I/R group, while single administration demonstrated limited efficacy. No significant differences in plasma antioxidants such as VC, urate and VE, or in the redox status of $\mathrm{CoQ}_{9}$, were observed among the four groups. In contrast, the plasma \%18:1 was significantly increased in the I/R group for the first 7 days as compared to the sham operation group (Fig. 3). The above results suggest that cellular oxidative damage in the rat brain is evident for at least 7 days after $I / R$ (Fig. 3). Repeated treatment suppressed the increase in \%18:1, whereas a single administration did not, which is consistent with the limited efficacy of single administration (Fig. 3).

\section{Edaravone as a Scavenger of Peroxynitrite}

The success of edaravone in the treatment of stroke prompted Dr. Hiide Yoshino at Yoshino Neurology Clinic in Chiba to use it as an ALS treatment since oxidative stress is believed to play an important role in this disease. After positive preliminary results, ${ }^{(21)}$ we and Dr. Yoshino began a collaboration in 2011 to determine the mechanism underlying the drug's efficacy. I believed our plasma oxidative markers would be useful in this investigation.

We recruited 26 ALS patients: 17 received edaravone $(30 \mathrm{mg}$ / day, 1-4 times a week) for at least 3 months, and 13 underwent this treatment regime for 6 months. ${ }^{(7)}$ Changes in the revised ALS functional rating scale (ALSFRS-R) were significantly smaller in the patients treated with edaravone than in the edaravoneuntreated ALS patients $(n=19)$ (Fig. 4). Based on the $\triangle$ ALSFRS-R results at 6 months $(\Delta)$, the patients were divided into 3 groups: a satisfactory progress group $(\Delta \geq 0)$, an ingravescent group $(\Delta<-5)$, and a middle group $(\Delta=-1 \sim-4)$. Fig. 4 shows that there were 6 patients in the satisfactory progress group of the 13 patients treated with edaravone for 6 months (or 7 of the 17 patients), but none out of the total of 19 untreated patients. Further, there were 3 patients in the ingravescent group among the total 13 edaravone-treated patients (or 4 out of 17 treated patients), but there were 12 out of a total of 19 untreated patients. These data indicate that edaravone treatment may have a significant beneficial outcome for ALS patients. ${ }^{(7)}$

Table 2 shows a comparison of plasma markers of circulatory oxidative stress and tissue oxidative damage between ALS patients and age-matched healthy controls. ${ }^{(7)}$ ALS patients had significantly increased plasma $\% \mathrm{CoQ}_{10}$ and $\%$ PUFA as compared to the controls, corroborating an increased level of oxidative stress and tissue oxidative damage in patients with ALS. However, administration of edaravone did not change $\% \mathrm{CoQ}_{10}$ and $\%$ PUFA (data not shown), indicating that the mechanism of action of edaravone does not depend on scavenging free radicals.

It is interesting that the plasma level of uric acid (UA) in ALS patients was significantly lower than that in the controls (Table 2) ${ }^{(7)}$ UA is a peroxynitrite scavenger, and thus this observation is consistent with increased cerebrospinal fluid levels of 3-

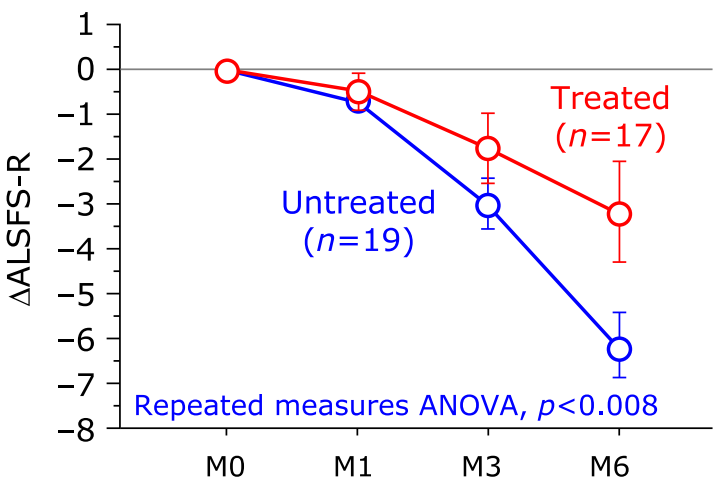

\begin{tabular}{|c|c|c|c|}
\hline Group & $\Delta$ & Untreated & Treated \\
\hline Satisfactory progress group & $\geq 0$ & 0 & $6(7) \uparrow$ \\
\hline Middle group & $-1 \sim-4$ & 7 & $4(6) \rightarrow$ \\
\hline Ingravescent group & $\leq-5$ & 12 & $3(4) \downarrow$ \\
\hline & Total & 19 & $13(17)$ \\
\hline
\end{tabular}

Fig. 4. Time course of changes of ALSFRS-R at 6 months with and without edaravone treatment (mean $\pm \mathrm{SE}$ ). Repeated-measures ANOVA shows a significant difference in $\triangle$ ALSFRS-R between the two groups $(p<0.008)$. Inset table provides a classification of the patients according to the value of $\triangle$ ALSFRS-R $(\Delta)$ at 6 months in edaravone-treated and -untreated groups of ALS patients. $\triangle$ ALSFRS-R at 6 months was missing for four patients in the treated group, and classifications were estimated on the assumption that $\triangle$ ALSFRS-R at 6 months was twice the $\triangle$ ALSFRS-R determination at 3 months, as indicated in parentheses.

Table 2. Levels of plasma antioxidants and lipids in patients with ALS as compared to age-matched healthy controls (average \pm SD)

\begin{tabular}{lccc}
\hline & ALS & Normal control & $p$ \\
\hline$n$ & 26 & 55 & \\
Male/Female & $14 / 12$ & $38 / 17$ & \\
Age & $60.9 \pm 11.2$ & $60.1 \pm 9.3$ & \\
VC, $\mu \mathrm{M}$ & $24.9 \pm 15.0$ & $31.1 \pm 21.0$ & \\
$\mathrm{UA}, \mu \mathrm{M}$ & $215 \pm 94$ & $314 \pm 93$ & $<0.001$ \\
$\mathrm{VE}, \mu \mathrm{M}$ & $33.5 \pm 10.2$ & $28.5 \pm 7.3$ & \\
$\mathrm{TQ} 10, \mathrm{nM}$ & $829 \pm 332$ & $710 \pm 206$ & \\
$\% \mathrm{CoQ}_{10}$ & $20.9 \pm 10.3$ & $3.9 \pm 1.3$ & $<0.001$ \\
FFA, $\mu \mathrm{M}$ & $486 \pm 377$ & $457 \pm 288$ & \\
$\% \mathrm{PUFA}$ & $19.0 \pm 4.1$ & $23.6 \pm 4.6$ & $<0.001$ \\
$\% 16: 1$ & $2.9 \pm 1.5$ & $3.9 \pm 1.4$ & \\
$\% 18: 1$ & $33.8 \pm 8.1$ & $34.4 \pm 5.1$ & \\
\hline
\end{tabular}

$P$ values were determined using the Student's $t$ test. VC, ascorbic acid $\mathrm{UA}$, uric acid; VE, vitamin $\mathrm{E} ; \mathrm{TQ} 10$, total coenzyme Q10; $\% \mathrm{CoQ}_{10}$, ratio of oxidized form of coenzyme Q10 to TQ10; FFA, free fatty acid; \%PUFA, ratio of polyunsaturated fatty acids to total FFA; $\% 16: 1$, ratio of palmitoleic acid to total FFA; $\% 18: 1$, ratio of oleic acid to total FFA.

nitrotyrosine observed in patients with ALS. ${ }^{(21-23)}$ Keizman et al. ${ }^{(24)}$ also reported low levels of serum UA in patients with ALS. Significantly, the UA levels in 36 out of 46 patients had decreased 6 months later, while the UA levels increased in 9 out of 46 patients and remained unchanged in one patient. ${ }^{(24)}$ Peroxynitrite is likely responsible for the prominent decrease in UA, since the VC and VE levels were similar in the control subjects (Table 2). ${ }^{(7)}$ Edaravone administration increased the plasma levels of UA in 10 out of 12 ALS patients (Fig. 5), and, notably, in 5 out of 5 subjects in 
Satisfactory progress group (A)
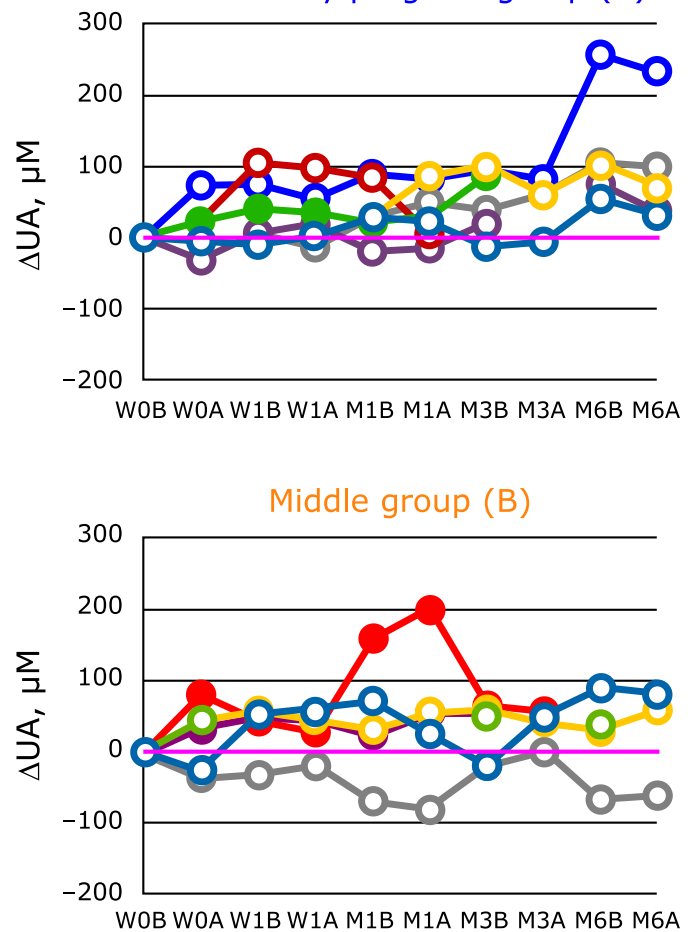

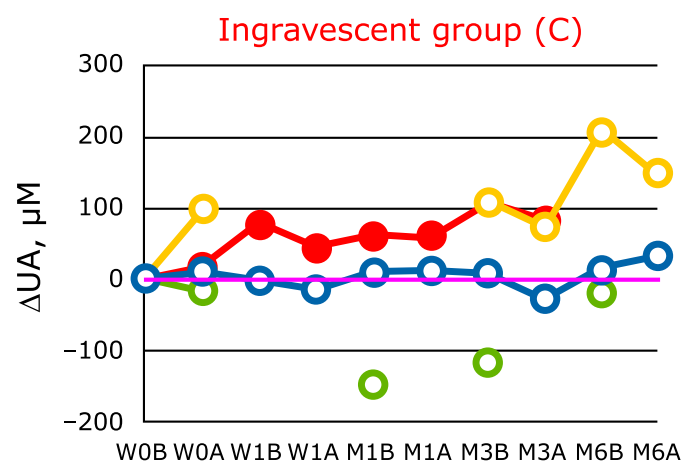

WOB WOA W1B W1A M1B M1A M3B M3A M6B M6A

Fig. 5. The increase or decrease in plasma urate levels, calculated from values at week 0 (before edaravone administration) and throughout the course of edaravone treatment in ALS patients. Patients were divided into a satisfactory progress group, an ingravescent group, and a middle group according to their values of $\triangle$ ALSFRS-R determined at 6 months of treatment. W0B/A and W1B/A are data for week 0 and week 1 before (B) and after (A) edaravone treatment, respectively; M1-6B/A are data for months 1-6 before (B) and after (A) edaravone treatment. Data for patients for whom the month 6 observation was missing are indicated by solid symbols.

the satisfactory progress group. ${ }^{(7)}$

We have also found that edaravone scavenges peroxynitrite approximately 30 times more efficiently than does UA. ${ }^{(25)}$ Little formation of 4-oxoedaravone and OPB was observed, suggesting that free radicals are not involved in the reaction of edaravone and peroxynitrite. ${ }^{(25)}$ Instead, the major product was 4-NO-edaravone, probably formed by the electrophilic addition of peroxynitrite and the removal of hydroperoxy anion $\left(\mathrm{HOO}^{-}\right) .{ }^{(25)}$ Moreover, Yoshino and Kimura ${ }^{(21)}$ previously reported that edaravone administration decreased 3-nitrotyrosine levels in the cerebrospinal fluid of ALS patients. These results are consistent with our contention that scavenging of peroxynitrite by edaravone may contribute significantly toward inhibiting the progression of ALS.

The Japanese government approved edaravone for the treatment of ALS patients on June 26, 2015. This will significantly benefit ALS patients because no other efficacious treatment is available. This development may be of interest to doctors who study other neurodegenerative diseases such as multiple sclerosis, ${ }^{(26,27)}$ multiple system atrophy, ${ }^{(28,29)}$ Parkinson's disease and Alzheimer's disease because the plasma urate levels of patients with these diseases are significantly lower than those of age-matched healthy controls. ${ }^{(29,30)}$

\section{References}

1 Sies H. Oxidative stress: introductory remarks. In: Sies H, ed. Oxidative Stress, London: Academic Press, 1985; 1-8.

2 Yamamoto Y, Kawamura M, Tatsuno K, et al. Formation of lipid hydroperoxides in the cupric ion-induced oxidation of plasma and low density lipoprotein. In: Davies KJA, ed. Oxidative Damage and Repair, New York: Pergamon Press, 1991; 287-291.

3 Bowry VW, Stocker R. Tocopherol-mediated peroxidation. The prooxidant effect of vitamin $\mathrm{E}$ on the radical-initiated oxidation of human low-density

\section{Abbreviations}

$\% 16: 1$

$\% 18: 1$

ALS

ALSFRS-R revised ALS functional rating scale

$\mathrm{CoQ}_{10}$

$\mathrm{CoQ}_{10} \mathrm{H}_{2}$

$\% \mathrm{CoQ}_{10}$

FFA

$\%$ PUFA

UA

$\mathrm{VC}$

$\mathrm{VE}$

percentage of palmitoleic acid in total free fatty acids percentage of oleic acid in total free fatty acids amyotrophic lateral sclerosis

oxidized form of coenzyme Q10

reduced form of coenzyme Q10

percentage of $\mathrm{CoQ}_{10}$ in total coenzyme Q10

free fatty acid

percentage of polyunsaturated fatty acid in total free fatty acids

uric acid

vitamin $\mathrm{C}$

vitamin $\mathrm{E}$

\section{Conflict of Interest}

No potential conflicts of interest were disclosed.

lipoprotein. J Am Chem Soc 1993; 115: 6029-6044.

4 Yamashita S, Yamamoto Y. Simultaneous detection of ubiquinol and ubiquinone in human plasma as a marker of oxidative stress. Anal Biochem 1997; 250: $66-73$.

5 Yamamoto Y, Yamashita S, Fujisawa A, Kokura S, Yoshikawa T. Oxidative stress in patients with hepatitis, cirrhosis, and hepatoma evaluated by plasma antioxidants. Biochem Biophys Res Commun 1998; 247: 166-170.

6 Miyamae T, Seki M, Naga T, et al. Increased oxidative stress and coenzyme 
Q10 deficiency in juvenile fibromyalgia: amelioration of hypercholesterolemia and fatigue by ubiquinol-10 supplementation. Redox Rep 2013; 18: 12-19.

7 Nagase M, Yamamoto Y, Miyazaki Y, Yoshino H. Increased oxidative stress in patients with amyotrophic lateral sclerosis and the effect of edaravone administration. Redox Rep 2016; 21: 104-112.

8 Hara K, Yamashita S, Fujisawa A, Ishiwa S, Ogawa T, Yamamoto Y. Oxidative stress in newborn infants with and without asphyxia as measured by plasma antioxidants and free fatty acids. Biochem Biophys Res Commun 1999; 257: 244-248.

9 Yamamoto Y, Sone H, Yamashita S, et al. Oxidative stress in LEC rats evaluated by plasma antioxidants and free fatty acids. J Trace Elem Exp Med 1997; 10: 129-134.

10 Yamamoto Y, Nagata Y, Katsurada M, Sato S, Ohori Y. Changes in rat plasma-free fatty acid composition under oxidative stress induced by carbon tetrachloride: decrease of polyunsaturated fatty acids and increase of palmitoleic acid. Redox Rep 1996; 2: 121-125.

11 Yamamoto Y, Yanagisawa M, Tak NW, et al. Repeated edaravone treatment reduces oxidative cell damage in rat brain induced by middle cerebral artery occlusion. Redox Rep 2009; 14: 251-258.

12 Yasuda M, Fujita T. Effect of lipid peroxidation on phospholipase $A_{2}$ activity of rat liver mitochondria. Jpn J Pharmacol 1977; 27: 429-435.

13 Weglicki WB, Dickens BF, Mak IT. Enhanced lysosomal phospholipid degradation and lysophospholipid production due to free radicals. Biochem Biophys Res Commun 1984; 124: 229-235.

14 Beckman JK, Borowitz SM, Burr IM. The role of phospholipase A activity in rat liver microsomal lipid peroxidation. J Biol Chem 1987; 262: 1479-1481.

15 Gutteridge JM, Quinlan GJ, Yamamoto Y. Hypothesis: are fatty acid patterns characteristic of essential fatty acid deficiency indicative of oxidative stress? Free Radic Res 1998; 28: 109-114.

16 Ntambi JM. Regulation of stearoyl-CoA desaturase by polyunsaturated fatty acids and cholesterol. J Lipid Res 1999; 40: 1549-1558.

17 Abe K, Yuki S, Kogure K. Strong attenuation of ischemic and postischemic brain edema in rats by a novel free radical scavenger. Stroke 1988; 19: 480485.

18 Nishi H, Watanabe T, Sakurai H, Yuki S, Ishibashi A. Effect of MCI-186 on brain edema in rats. Stroke 1989; 20: 1236-1240.
19 Yamamoto Y, Kuwahara T, Watanabe K, Watanabe K. Antioxidant activity of 3-methyl-1-phenyl-2-pyrazolin-5-one. Redox Rep 1996; 2: 333-338.

20 Edaravone Acute Infarction Study Group. Effect of a novel free radical scavenger, edaravone (MCI-186), on acute brain infarction. Randomized, placebocontrolled, double-blind study at multicenters. Cerebrovasc Dis 2003; 15: 222-229.

21 Yoshino H, Kimura A. Investigation of the therapeutic effects of edaravone, a free radical scavenger, on amyotrophic lateral sclerosis (Phase II study). Amyotroph Lateral Scler 2006; 7: 241-245.

22 Beal MF, Ferrante RJ, Browne SE, Matthews RT, Kowall NW, Brown RH Jr. Increased 3-nitrotyrosine in both sporadic and familial amyotrophic lateral sclerosis. Ann Neurol 1997; 42: 644-654.

23 Tohgi H, Abe T, Yamazaki K, Murata T, Ishizaki E, Isobe C. Remarkable increase in cerebrospinal fluid 3-nitrotyrosine in patients with sporadic amyotrophic lateral sclerosis. Ann Neurol 1999; 46: 129-131.

24 Keizman D, Ish-Shalom M, Berliner S, et al. Low uric acid levels in serum of patients with ALS: further evidence for oxidative stress? J Neurol Sci 2009; 285: 95-99.

25 Fujisawa A, Yamamoto Y. Edaravone, a potent free radical scavenger, reacts with peroxynitrite to produce predominantly the 4-NO-edaravone. Redox Rep 2016; 21: 98-103.

26 Hooper DC, Spitsin S, Kean RB, et al. Uric acid, a natural scavenger of peroxynitrite, in experimental allergic encephalomyelitis and multiple sclerosis. Proc Natl Acad Sci U S A 1998; 95: 675-680.

27 Toncev G, Milicic B, Toncev S, Samardzic C. Serum uric acid levels in multiple sclerosis patients correlate with activity of disease and blood-brain barrier dysfunction. Eur J Neurol 2002; 9: 221-226.

28 Cao B, Guo X, Chen K, et al. Uric acid is associated with the prevalence but not disease progression of multiple system atrophy in Chinese population. $J$ Neurol 2013; 260: 2511-2515.

29 Chen D, Wei X, Zou J, et al. Contra-directional expression of serum homocysteine and uric acid as important biomarkers of multiple system atrophy severity: a cross-sectional study. Front Cell Neurosci 2015; 9: 247.

30 Kim TS, Pae CU, Yoon SJ, et al. Decreased plasma antioxidants in patients with Alzheimer's disease. Int J Geriatr Psychiatry 2006; 21: 344-348. 